\title{
Fatores associados com a dificuldade no acesso de idosos com deficiência aos serviços de saúde
}

\author{
Factors associated with difficulty of access of the elderly \\ with disabilities to the health services
}

Fabienne Louise Juvêncio dos Santos Amaral ${ }^{1}$

Márcia Heloyse Alves Motta ${ }^{1}$

Laíla Pereira Gomes da Silva ${ }^{1}$

Simone Bezerra Alves ${ }^{1}$

${ }^{1}$ Departamento de

Fisioterapia, Centro de

Ciências da Saúde,

Universidade Federal da

Paraíba. Cidade

Universitária, Campus I.

58051-900 João Pessoa

PB.simonea@gmail.com

\begin{abstract}
This study seeks to analyze which are the variables associated with the difficulty of elderly people with disabilities gaining access to the health services. This is an observational study of an analytical cross-sectional nature, with a sample of 244 elderly people with disabilities. Data relating to socio-economic profile, the nature of the disability, and the conditions of access to health services were gathered. Version 11.0 of the Statistical Package for the Social Sciences software was used for descriptive, statistical and analytical assessment of the data. The protection variables for difficulties in being treated in the health services were: the lack of drains, culverts, trash, bags of refuse, or irregular floor surfaces; the absence of ramps on sidewalks and pavements; the availability of transport; ease in scheduling appointments; and the length of the waiting period to be attend$e d$. The number of factors listed shows that the architectonic barriers and the current situation of healthcare need to be adequate in order to ensure full access and use by the elderly with disabilities to the health services.
\end{abstract}

Key words Aging, People with disabilities, Access to the health services
Resumo Este estudo propõe analisar quais variáveis se associam com a dificuldade no acesso de idosos com deficiência aos serviços de saúde. Trata-se de um estudo observacional analitico de caráter transversal, com uma amostra de 244 idosos com deficiência. Foram agrupados dados pertinentes ao perfil socioeconômico, à caracterização da deficiência e às condições de acessibilidade aos serviços de saúde. Para análise estatística descritiva e analitica dos dados, foi utilizado o software Statistical Package for the Social Science, versão 11.0. As variáveis de proteção para a dificuldade em ser atendido nos serviços de saúde foram: ausência de esgotos, bueiros, dejetos, sacos de lixos, ou pisos quebrados; ausência de degraus nas calçadas e existência de calçamento; presença de transporte; facilidade na marcação de consultas; e o baixo tempo de espera para ser atendido. O somatório dos fatores expostos mostra que as barreiras arquitetônicas e situação atual de atenção à saúde precisam ser adequadas, visando o ingresso e utilização plena dos idosos com deficiência aos serviços de saúde.

Palavras-chave Envelhecimento, Pessoas com deficiência, Acesso aos serviços de saúde 


\section{Introdução}

O envelhecimento populacional é uma realidade recente para o Brasil, assim como para os demais países em desenvolvimento ${ }^{1}$. Estima-se que existam 17,6 milhões de idosos no país e que, para o ano de 2050, sejam cerca de dois bilhões de pessoas com 60 anos ou mais no mundo ${ }^{2}$. No entanto, sabe-se que o processo de envelhecimento em situações de doenças, principalmente crônico-degenerativas, associadas a fatores externos, como hábitos de vida, pode desencadear uma condição de deficiência que requeira assistência ${ }^{3}$. Assim, segundo o recente relatório mundial sobre a deficiência, as populações vulneráveis, como os idosos, são afetadas desproporcionalmente pela deficiência ${ }^{4}$.

O conceito de deficiência está relacionado com a perda ou anormalidade de uma estrutura ou função psicológica, fisiológica ou anatômica que pode gerar incapacidade ou dificuldade para o desempenho de uma atividade, dentro do padrão considerado normal para o ser humano ${ }^{5}$. Porém, sabe-se que esse é um conceito em evolução e resultante da interação entre as pessoas com limitações e as barreiras atitudinais e ambientais, que podem impedir a plena e efetiva participação dessas pessoas na sociedade em igualdade de oportunidades com as demais 6 .

A prevalência de pessoas com deficiência deverá aumentar com o crescimento da expectativa de vida ${ }^{7}$. Assim, o envelhecimento populacional tende a evidenciar a deficiência como uma condição circunstancial de tal fase da vida. Essa ideia torna o envelhecimento e a deficiência temas de interesse da sociedade em geral, e não mais limitado aos movimentos de pessoas com deficiência na busca de garantia dos direitos e efetividade das ações políticas ${ }^{8}$.

Atualmente, observa-se que, em algumas regiões, a assistência à saúde ainda está muito restrita aos serviços de atenção secundária, o que compromete a acessibilidade da população face à demanda reprimida nesses locais?. No intuito de melhorar o acesso da população à saúde, as Unidades de Saúde da Família (USF), através dos serviços no nível primário de atenção, objetivam estar qualificadas para atender e resolver os principais problemas que demandam os serviços de saúde ${ }^{10}$. Porém, de acordo com Frenk ${ }^{11}$, a acessibilidade pode ser definida não só como o grau de ajuste entre as características dos recursos de atenção à saúde, mas também como as características das populações, no processo de busca e obtenção da atenção em saúde.
Um recente estudo indica a importância da educação em saúde para combater as elevadas taxas de mortalidade e o desenvolvimento de deficiências na população idosa de países subdesenvolvidos da América Latina, Índia e China ${ }^{12}$. Prevenir a deficiência deve ser visto como uma estratégia multidimensional que abrange aspectos de prevenção das barreiras incapacitantes, assim como a precaução e o tratamento dos problemas de saúde subjacentes ${ }^{13}$. Sendo assim, ações preventivas de saúde devem ser desenvolvidas com o intuito de evitar o agravamento de doenças crônicas que possam levar a deficiências, com subsequente redução dos índices de mortalidade da população idosa.

No entanto, ainda há escassez de conhecimento sobre como a população idosa com deficiência efetivamente se beneficia pelas políticas públicas de saúde 9 . Assim, o presente estudo se propõe a analisar algumas variáveis que se associam à dificuldade no acesso de idosos com deficiência aos serviços de saúde.

\section{Metodologia}

Trata-se de um estudo observacional analítico de caráter transversal, realizado na cidade de João Pessoa (PB), de fevereiro à dezembro de 2008. A população de referência do estudo foi constituída por idosos com deficiência ou mobilidade reduzida, com idade acima dos 60 anos e de ambos os sexos.

Esta pesquisa faz parte de um estudo maior que avaliou a acessibilidade de pessoas com deficiência no município de João Pessoa (PB). Para este estudo maior, a amostra populacional foi calculada em 502 pessoas com deficiência ou mobilidade reduzida, considerando: dados da população ( $\mathrm{N}=561.894$ habitantes), de acordo com o Censo Demográfico $2000^{10}$; erro amostral máximo 1,4\% (e = 0,014); nível de confiança de $99 \%,(z=2,58)$; proporção favorável inicial estimada $(p=0,015)$, ou seja, $15 \%$ da população com deficiência; proporção não favorável inicial estimada $(q=0,985)$. Ao término da coleta dos dados foram obtidas 526 entrevistas.

A amostra populacional deste estudo constituiu-se de 244 idosos que atenderam aos critérios de inclusão: ter 60 anos ou mais, possuir alguma deficiência e/ou restrição de mobilidade e aceitar participar da pesquisa. Foram excluídos os indivíduos que se recusaram a responder a alguma das perguntas, possuíram dificuldade na compreensão das perguntas ou desistiram de participar do estudo. 
Para o acesso e seleção da amostra foram utilizados os cadastros dos idosos com deficiência ou mobilidade reduzida das Unidades de Saúde da Família (USF), envolvidas na pesquisa a partir de uma triagem com base na divisão dos cinco Distritos Sanitários existentes. Estes são as unidades operacionais e administrativas mínimas do sistema de saúde, definida com critérios geográficos, populacionais, epidemiológicos, administrativos e políticos ${ }^{14}$. Assim, fez-se a separação dos Distritos por estratos, e a partir de cada um formou-se conglomerados, dos quais foram sorteadas aleatoriamente 30 USF: 7 do Distrito I; 8 do Distrito II; 6 do Distrito III; 7 do Distrito IV; 2 do Distrito V. Como a área de abrangência destes são diferentes, procurou-se dividir a amostra proporcionalmente.

Utilizou-se, como instrumento, um formulário estruturado. Neste foram agrupados dados pertinentes ao perfil socioeconômico; caracterização da deficiência; e às condições de acessibilidade aos serviços de saúde, abordando aspectos que podem dificultar tal acesso.

A coleta de dados consistiu de entrevistas domiciliares, realizadas por 17 estudantes do Centro de Ciências da Saúde da Universidade Federal da Paraíba (UFPB), que foram devidamente treinados para a utilização do instrumento.

As primeiras análises dos dados foram de ordem descritiva e exploratória, a fim de ter uma referência base para as variáveis em estudo. Empregou-se o coeficiente de Alpha de Cronbach para verificar a confiabilidade do instrumento, obtendo-se coeficiente 0,67 e 0,82 para as dimensões perfil socioeconômico e acesso aos serviços de saúde, respectivamente.

Os dados obtidos foram tabulados utilizando-se o software Statistical Package for the Social Science (SPSS) versão 11.0. Os mesmos foram submetidos à análise estatística descritiva e analítica, adotando-se um nível de significância de $\mathrm{p}$ $<0,05$. Na análise descritiva fez-se a distribuição das frequências absolutas e relativas.

$\mathrm{Na}$ abordagem analítica foi realizada análise bivariada utilizando o teste do qui-quadrado de Pearson $\left(\chi^{2}\right)$, para se observarem as possíveis associações existentes entre as variáveis independentes e as dependentes. Em seguida foi realizada análise multivariada, por regressão logística binária, adotando método backward stepwise. Todas as variáveis independentes foram incluídas no modelo inicial e então foram regressivamente excluídas até que somente aquelas com valor $\mathrm{p}$ inferior a 0,05 foram mantidas, com cálculo das OR ajustadas.
Este estudo foi submetido à apreciação do Comitê de Ética em Pesquisa do Centro de Ciências da Saúde da UFPB, de acordo com as atribuições da Resolução no. $196 / 96^{15}$ do Conselho Nacional de Saúde.

\section{Resultados}

A amostra foi composta por 244 idosos, com predomínio de indivíduos do sexo feminino $(63,5 \%)$ e faixa etária de 80 anos ou mais. Destacaram-se os idosos viúvos $(37,3 \%)$ e que possuíam baixo nível de escolaridade (36,5\% eram alfabetizados e $35,2 \%$ analfabetos). Verificou-se que a maioria dos idosos (82,0\%) não possuía plano de saúde privado e possuía renda de 1 salário mínimo (60,2\%). As demais características socioeconômicas são apresentadas na Tabela 1 .

Tabela 1. Distribuição dos idosos com deficiência segundo aspectos socioeconômicos. João Pessoa, PB, 2008.

\begin{tabular}{|c|c|c|}
\hline \multirow[b]{2}{*}{ Variáveis } & \multicolumn{2}{|c|}{ Frequências } \\
\hline & $\mathbf{n}$ & $\%$ \\
\hline \multicolumn{3}{|l|}{ Sexo } \\
\hline Feminino & 155 & 63,5 \\
\hline Masculino & 89 & 36,5 \\
\hline \multicolumn{3}{|l|}{ Idade (anos) } \\
\hline $60-64$ & 42 & 17,2 \\
\hline $65-69$ & 51 & 20,9 \\
\hline $70-74$ & 29 & 11,9 \\
\hline $75-79$ & 44 & 18,0 \\
\hline 80 ou mais & 78 & 32,0 \\
\hline \multicolumn{3}{|l|}{ Situação Conjugal } \\
\hline Casado/em união & 86 & 35,2 \\
\hline Solteiro & 47 & 19,3 \\
\hline Viúvo & 91 & 37,3 \\
\hline Separado/divorciado & 20 & 8,2 \\
\hline \multicolumn{3}{|l|}{ Escolaridade } \\
\hline Analfabeto & 86 & 35,2 \\
\hline Alfabetizado & 89 & 36,5 \\
\hline Ens. Fundamental Completo & 45 & 18,4 \\
\hline Ens. Médio Completo & 18 & 7,4 \\
\hline Ens. Superior Completo & 6 & 2,5 \\
\hline \multicolumn{3}{|l|}{ Plano de Saúde Privado } \\
\hline $\operatorname{Sim}$ & 44 & 18,0 \\
\hline Não & 200 & 82,0 \\
\hline \multicolumn{3}{|l|}{ Renda Pessoal } \\
\hline Não possui renda & 15 & 6,1 \\
\hline$<1$ salário mínimo & 3 & 1,2 \\
\hline 1 salário mínimo & 147 & 60,2 \\
\hline 1-2 salários mínimos & 50 & 20,5 \\
\hline 2-5 salários mínimos & 26 & 10,7 \\
\hline > 5 salários mínimos & 3 & 1,2 \\
\hline
\end{tabular}


Em relação à caracterização da deficiência (Tabela 2), constatou-se que a de maior ocorrência foi a física $(49,2 \%)$, seguida da mobilidade reduzida $(20,5 \%)$, deficiência mental $(11,5 \%)$, visual $(11,5 \%)$, auditiva $(6,6 \%)$ e múltipla $(0,8 \%)$. Além disto, 28,7\% dos idosos afirmaram ter adquirido a deficiência entre 0 e 4 anos antes da entrevista e 73,8\% necessitavam usar algum tipo de tecnologia assistiva.

Concernente aos tipos de barreiras arquitetônicas que dificultaram a acessibilidade dos idosos com deficiência aos serviços de saúde, destacaram-se: ausência de transporte $(8,2 \%)$; existência de esgotos, bueiros, dejetos, sacos de lixos, ou pisos quebrados $(4,1 \%)$; e presença de degraus nas calçadas e inexistência de calçamento $(3,7 \%)$ (Tabela 3$)$. Os resultados podem ser observados na Tabela 3.

$\mathrm{Na}$ Tabela 4 são apresentadas as limitações dos serviços de atenção à saúde. Observou-se que as principais limitações referidas pelos idosos foram: ausência de atendimento prioritário $(38,9 \%)$; elevado tempo de espera para ser atendido $(27,9 \%)$; inexistência de atendimento domiciliar de profissionais da saúde $(21,3 \%)$; dificuldade em marcar consultas $(11,9 \%)$; e inexistência da visita domiciliar do Agente Comunitário de Saúde (9,0\%).

Tabela 2. Distribuição dos idosos segundo caracterização da deficiência. João Pessoa, PB , 2008.

\begin{tabular}{lcr}
\hline & \multicolumn{2}{c}{ Frequências } \\
\cline { 2 - 3 } \multicolumn{1}{c}{ Variáveis } & $\mathbf{n}$ & $\%$ \\
\hline Tipo de deficiência & & \\
Mental & 28 & 11,5 \\
Visual & 28 & 11,5 \\
Física & 120 & 49,2 \\
Auditiva & 16 & 6,6 \\
Múltipla & 2 & 0,8 \\
Mobilidade Reduzida & 50 & 20,5 \\
Tempo da deficiência (anos) & & \\
0-4 & 70 & 28,7 \\
$5-9$ & 60 & 24,6 \\
10-19 & 40 & 16,4 \\
20-29 & 16 & 6,6 \\
$30-39$ & 14 & 5,7 \\
$40-49$ & 7 & 2,9 \\
50 ou mais & 25 & 10,2 \\
Não soube informar & 12 & 4,9 \\
Necessidade do uso de tecnologia & & \\
assistiva & & \\
Sim & 180 & 73,8 \\
Não & 64 & 26,2 \\
\hline
\end{tabular}

Tabela 3. Prevalência de barreiras arquitetônicas que dificultaram a acessibilidade aos serviços de saúde, segundo os idosos com deficiência. João Pessoa, PB, 2008.

\begin{tabular}{lrr}
\hline & \multicolumn{2}{c}{ Frequências } \\
\cline { 2 - 3 } \multicolumn{1}{c}{ Variáveis } & $\mathbf{n}$ & $\%$ \\
\hline Existência de esgotos, bueiros, dejetos, & & \\
sacos de lixos, ou pisos quebrados & & \\
$\quad$ Sim & 10 & 4,1 \\
$\quad$ Não & 234 & 95,9 \\
Presença de degraus nas calçadas e & & \\
inexistência de calçamento & & \\
$\quad$ Sim & 9 & 3,7 \\
$\quad$ Não & 235 & 96,3 \\
Falta de sinais de trânsito & & \\
$\quad$ Sim & 2 & 0,8 \\
$\quad$ Não & 242 & 99,2 \\
Falta de adaptação nos transportes & & \\
$\quad$ Sim & 5 & 2,0 \\
$\quad$ Não & 239 & 98,0 \\
Ausência de transporte & & \\
$\quad$ Sim & 20 & 8,2 \\
$\quad$ Não & 224 & 91,8 \\
\hline
\end{tabular}

Tabela 4. Limitações e potencialidade dos serviços de atenção à saúde, segundo os idosos com deficiência. João Pessoa, PB , 2008.

\begin{tabular}{|c|c|c|}
\hline \multirow[b]{2}{*}{ Variáveis } & \multicolumn{2}{|c|}{ Frequências } \\
\hline & $\mathbf{n}$ & $\%$ \\
\hline \multicolumn{3}{|c|}{ Dificuldade em marcar consultas } \\
\hline Sim & 29 & 11,9 \\
\hline Não & 215 & 88,1 \\
\hline \multicolumn{3}{|l|}{ Ausência de especialistas } \\
\hline $\operatorname{Sim}$ & 7 & 2,9 \\
\hline Não & 237 & 97,1 \\
\hline \multicolumn{3}{|l|}{ Atendimento prioritário } \\
\hline Sim & 149 & 61,1 \\
\hline Não & 95 & 38,9 \\
\hline \multicolumn{3}{|c|}{$\begin{array}{l}\text { Elevado tempo de espera para ser } \\
\text { atendido }\end{array}$} \\
\hline Sim & 68 & 27,9 \\
\hline Não & 176 & 72,1 \\
\hline \multicolumn{3}{|c|}{$\begin{array}{l}\text { Atendimento domiciliar de } \\
\text { profissionais da saúde }\end{array}$} \\
\hline Sim & 192 & 78,7 \\
\hline Não & 52 & 21,3 \\
\hline \multicolumn{3}{|c|}{ Visita domiciliar do Agente } \\
\hline \multicolumn{3}{|c|}{ Comunitário de Saúde } \\
\hline Sim & 222 & 91,0 \\
\hline Não & 22 & 9,0 \\
\hline \multicolumn{3}{|c|}{ Preconceito dos profissionais } \\
\hline $\operatorname{Sim}$ & 1 & 0,4 \\
\hline Não & 243 & 99,6 \\
\hline
\end{tabular}


$\mathrm{Na}$ análise bivariada (Tabela 5), as seguintes variáveis apresentaram associação estatisticamente significante com a dificuldade em ser atendido nos serviços de saúde: Existência de esgotos, bueiros, dejetos, sacos de lixos, ou pisos quebrados; presença de degraus nas calçadas e inexistência de calçamento; falta de adaptação nos transportes; ausência de transporte; dificuldade em marcar consultas; ausência de especialistas; ausência de atendimento prioritário; elevado tempo de espera para ser atendido; e inexistência de visita domiciliar do Agente Comunitário de Saúde. Não houve valores estatisticamente significantes para os aspectos socioeconômicos e caracterização da deficiência.

Por meio da análise multivariada, observouse que os fatores de proteção para a dificuldade em ser atendido nos serviços de saúde (Tabela 5) foram: ausência de esgotos, bueiros, dejetos, sacos de lixos, ou pisos quebrados; ausência de degraus nas calçadas e existência de calçamento; presença de transporte; facilidade na marcação de consultas; e o baixo tempo de espera para ser atendido.

\section{Discussão}

As pessoas idosas são mais acometidas por doenças crônicas, com menor exposição a acidentes, homicídios e consumo de drogas ${ }^{16,17}$. E essas doenças, por sua vez, podem levar a faixa etária de idosos com idade avançada ( 80 anos ou mais) ao desenvolvimento de deficiências, o que foi constatado no presente estudo. Os nossos achados corroboram com os resultados de Caldas ${ }^{16}$, ao

Tabela 5. Análise bruta e multivariada para associação das variáveis independentes em relação ao desfecho dificuldade em ser atendido nos serviços de saúde.

\begin{tabular}{|c|c|c|c|c|}
\hline \multirow[b]{2}{*}{ Variáveis } & \multicolumn{2}{|c|}{ Análise bruta } & \multicolumn{2}{|c|}{ Análise ajustada } \\
\hline & RP (IC95\%) & p & RP (IC95\%) & $\mathrm{p}$ \\
\hline $\begin{array}{l}\text { Existência de esgotos, bueiros, dejetos, sacos de } \\
\text { lixos, ou pisos quebrados }\end{array}$ & & 0,000 & & 0,002 \\
\hline $\operatorname{Sim}$ & 1,00 & & 1,00 & \\
\hline Não & $0,88[0,80-0,96]$ & & $0,02[0,01-0,28]$ & \\
\hline $\begin{array}{l}\text { Presença de degraus nas calçadas e inexistência de } \\
\text { calçamento }\end{array}$ & & 0,001 & & 0,014 \\
\hline Sim & 1,00 & & 1,00 & \\
\hline Não & $0,91[0,84-0,98]$ & & $0,08[0,01-0,59]$ & \\
\hline Falta de adaptação nos transportes & & 0,001 & & * \\
\hline $\operatorname{Sim}$ & 1,00 & & & \\
\hline Não & $0,93[0,87-0,99]$ & & * & \\
\hline Ausência de transporte & & 0,000 & & 0,000 \\
\hline $\operatorname{Sim}$ & 1,00 & & 1,00 & \\
\hline Não & $0,76[0,66-0,87]$ & & $0,03[0,01-0,20]$ & \\
\hline Dificuldade em marcar consultas & & 0,000 & & 0,000 \\
\hline Sim & 1,00 & & 1,00 & \\
\hline Não & $0,62[0,51-0,74]$ & & $0,02[0,01-0,05]$ & \\
\hline Ausência de especialistas & & 0,001 & & * \\
\hline Sim & 1,00 & & & \\
\hline Não & $0,92[0,86-0,99]$ & & * & \\
\hline Atendimento prioritário & & 0,000 & & * \\
\hline Sim & 1,00 & & & \\
\hline Não & $2,02[1,50-2,71]$ & & * & \\
\hline Elevado tempo de espera para ser atendido & & 0,000 & & 0,001 \\
\hline $\operatorname{Sim}$ & 1,00 & & 1,00 & \\
\hline Não & $0,64[0,51-0,81]$ & & $0,23[0,10-0,55]$ & \\
\hline Visita domiciliar do Agente Comunitário de Saúde & & 0,008 & & * \\
\hline Sim & 1,00 & & & \\
\hline Não & $2,81[1,27-6,21]$ & & * & \\
\hline
\end{tabular}

* Removida da análise devido à perda de significância estatística na análise ajustada. 
afirmar que a alteração demográfica mais importante que influencia no aumento da frequência na utilização dos serviços de saúde é o rápido crescimento da proporção de pessoas com mais de 85 anos. Esses resultados sugerem que idosos com idade mais avançada estão mais suscetíveis ao aparecimento de situações patológicas que os levem à deficiência. Essa distribuição pode estar intimamente ligada ao aumento da morbidade em idades mais elevadas, ocasionando no idoso um período de dependência e necessidade de cuidados ${ }^{17}$. Destaca-se ainda que as alterações biológicas que ocorrem no envelhecimento diminuem a capacidade de manutenção da homeostase quando existe exposição a algum fator de estresse, que, por sua vez, pode levar a desenvolvimento de deficiências ${ }^{3}$.

Os dados referentes aos sujeitos com deficiência que se encontravam na sexagésima década de vida também foram semelhantes aos encontrados na literatura ${ }^{17-19}$, representando $38,1 \%$ da amostra. Observou-se maior ocorrência de idosos do sexo feminino $(63,5 \%)$ que se assemelham aos dados achados de Garcia e Saintrain ${ }^{20}$, confirmando o processo de feminilização da população idosa com deficiência. Mulheres buscam atendimento com maior frequência do que homens. O mesmo aspecto foi observado com pessoas com deficiência em investigações da Organização Mundial da Saúde independente da condição socioeconômica e da idade, seja em países desenvolvidos ou subdesenvolvidos ${ }^{4}$.

Apesar das idosas apresentarem uma maior esperança de vida, também possuem maior proporção de anos vividos com doenças ${ }^{21}$. Assim, principalmente as mulheres idosas, apresentam declínio da capacidade funcional mais acentuada, resultando em perda da autonomia e dificuldade de realização de suas atividades cotidianas.

Além das doenças e incapacidades frequentes nessa faixa etária, que tornam a saúde precária, há a associação de outros indicadores, como a relação entre baixo nível de escolaridade e renda e a carência de saneamento básico, que contribuem para essa precarização ${ }^{22}$. A literatura aponta que houve uma diminuição da associação descrita anteriormente, elencando três hipóteses ${ }^{23}$ : (1) redução do desnível da renda adquirida após a aposentadoria; (2) promoção de maior acesso aos serviços, por meio de políticas sociais; (3) viés de sobrevivência (idosos com situação socioeconômica mais desfavorável, tendem para um óbito precoce) e/ou viés em virtude de não-participação (exclusão da participação dos estudos de base populacional dos idosos institucionalizados).
Entretanto, o baixo nível de escolaridade constatado reflete as condições educacionais precárias do início do século $\mathrm{XX}$, o que repercute no cotidiano do idoso com deficiência, como no manuseio de medicamentos, prescrições e dietas, que em caso de uso inadequado acarreta prejuízos para a sua saúde ${ }^{18,20}$. Somado a isso, é sabido que pessoas que vivem em situação econômica precária estão mais expostas ao risco de adoecer e morrer, quadro este que se intensifica em populações mais desprotegidas ou vulneráveis, como crianças, idosos e deficientes ${ }^{19}$.

No presente estudo, observou-se precariedade das condições socioeconômicas dos idosos com deficiência, visto que $67,5 \%$ ganhavam até 1 salário mínimo e $82,0 \%$ não possuíam plano de saúde privado. Neste estudo apenas a minoria dos entrevistados utilizava a rede privada de assistência à saúde, fator este que pode estar diretamente relacionado com uma menor renda e nível educacional dos entrevistados ${ }^{18}$. Diferentemente do processo de envelhecimento vivenciado pelos países europeus, permeado pelo desenvolvimento social e aumento de renda, não só o Brasil, mas vários países latino-americanos enfrentam o envelhecimento da população envolto por um processo de urbanização precário e pouca alteração da distribuição de renda ${ }^{17}$. De acordo com alguns autores, esse desnível socioeconômico em relação aos países europeus, de certa maneira, poderia ser compensado pelo suporte familiar aos idosos ${ }^{17,24}$.

Vários registros da literatura concordam que pessoas com deficiência enfrentam condições socioeconômicas inferiores que aquelas sem deficiência. Assim, esses dados corroboram com o presente estudo, uma vez que mesmo sem haver comparação entre pessoas com e sem deficiência, podese constatar elevados níveis de pobreza, com baixas taxas de emprego e baixo nível de escolaridade entre as pessoas com deficiência. Estas também têm acesso distintos aos serviços de saúde e, portanto, possuem necessidades não satisfeitas, quando comparadas à população em geral ${ }^{25-28}$.

Sabe-se que durante o processo de envelhecimento observam-se alterações biológicas ${ }^{29}$. Assim essa faixa etária está mais predisposta a acometimentos por doenças crônicas, que geralmente são enfermidades complexas e bastante onerosas por necessitar de cuidados e exames contínuos e periódicos ${ }^{30,31}$. A progressão das alterações naturais, se não acompanhadas, pode resultar em incapacidades funcionais e deficiências transitórias ou permanentes. Assim, tais modificações, associadas com a carência de programas ou ações 
preventivas para o desenvolvimento de doenças crônicas, podem contribuir para o incremento de deficiências na população estudada.

Estudo prévio sobre a acessibilidade de pessoas com deficiência, realizado por nosso grupo, constatou que em população maior de 18 anos, incluindo idosos, há grande prevalência de pessoas com deficiência física $(40 \%)$ e mobilidade reduzida (15,3\%), entretanto em menor proporção que quando isolada a amostra de idosos (respectivamente $49,2 \%$ e $20,5 \%)^{32}$. Tal fato pode estar relacionado com o aparecimento de doenças crônico-degenerativas, como diabetes, hipertensão, obesidade, doenças reumáticas, doenças cardiovasculares, entre outras comuns nessa população ${ }^{30}$. Essas patologias possuem um longo período de evolução e provocam limitações funcionais, restrição de movimentos e dificuldades de deambulação ${ }^{33}$.

Observou-se que 11,5\% dos idosos apresentaram deficiência mental e esta elevada prevalência pode estar diretamente relacionada ao aumento da expectativa de vida e ao aparecimento de doenças neuro-degenerativas ${ }^{34}$. Entretanto, tais dados são mais prevalentes quando considerados os maiores de 18 anos, uma vez que Amaral et al. ${ }^{32}$ constataram que $30 \%$ da amostra de pessoas com deficiência a apresentava como mental. Considerando-se os dados das pessoas idosas, sabe-se que a doença de Alzheimer é a causa mais comum de deficiência mental e demência, de acordo com estudo realizados em São Paulo ${ }^{35}$. Coelho et al. $^{34}$ correlacionaram o sexo feminino e a baixa escolaridade como fatores de maior predisposição para o surgimento das demências, o que é coerente com a predominância do sexo feminino e o baixo nível de escolaridade da população estudada.

Em relação às alterações sensoriais, identificou-se que $11,5 \%$ dos idosos possuíam deficiência visual e 6,6\% deficiência auditiva, sendo congênitas ou decorrentes do processo de envelhecimento. Sabe-se que as mudanças visuais podem também estar relacionadas com patologias associadas, como a catarata, o glaucoma e a diabetes mellitus $^{33}$. Essas condições podem acarretar o isolamento e a perda de motivação e de participação do convívio social.

Referente ao tempo da deficiência, observouse que $28,7 \%$ dos idosos diagnosticaram suas deficiências em até 4 anos antes da realização da entrevista e $24,6 \%$ entre 5 e 9 anos, totalizando um porcentual de mais da metade da amostra $(53,3 \%)$. Considerando que aproximadamente $60 \%$ da população estudada possuía faixa etária superior a 70 anos, infere-se que o aparecimento tardio da deficiência pode ser decorrente do surgimento de algumas patologias incapacitantes associadas ao envelhecimento, como a osteoporose ${ }^{36}$.

Observou-se, também, que para $25,4 \%$ da amostra estudada, o surgimento da deficiência ocorreu em período superior a 20 anos. Assim, este estudo enfatiza também a existência da deficiência como um fator que pode ter dificultado a inserção do indivíduo em atividades educacionais e no mercado de trabalho, impossibilitando, assim, a obtenção de uma melhor condição socioeconômica e, consequentemente, melhor qualidade de vida e cuidado com sua saúde.

Dos idosos entrevistados, 73,8\% referiu usar algum dispositivo assistivo a fim de minimizar sua deficiência. Sabe-se que as alterações sofridas no envelhecimento e suas consequências podem fazer com que seja necessária a utilização de dispositivos que auxiliem na retomada da função e da autonomia do idoso. Portanto, adotase a alternativa de tecnologia assistiva para alcançar tais objetivos, pois se entende que essa tecnologia, segundo a Classificação Internacional de Funcionalidade, Incapacidade e Saúde, é representada por "qualquer produto, instrumento, equipamento ou tecnologia adaptado ou especialmente projetado para melhorar a funcionalidade de uma pessoa incapacitada" ${ }^{37}$.

A implementação da Estratégia de Saúde da Família, que se iniciou em meados dos anos 90, objetivou contribuir para a construção e a consolidação do Sistema Único de Saúde (SUS). A mesma visa garantir, assim, uma mudança para o modelo assistencial a partir da atenção básica, priorizando, entre outros princípios, a integralidade nas práticas assistenciais, na qual a atenção do profissional apreenda as necessidades mais abrangentes do indivíduo, entre eles os idosos ${ }^{38}$. Porém, os locais de atendimento, geralmente, são inacessíveis, superlotados e sem adequada infraestrutura. As barreiras arquitetônicas impedem o exercício do mais básico dos direitos de qualquer cidadão, o de deslocar-se livremente ${ }^{39}$. Devido a essas barreiras, as ações de promoção e prevenção à saúde normalmente ficam restritas a algumas campanhas de vacinação, controle de hipertensão e diabetes ${ }^{30}$.

A saúde também é afetada por fatores ambientais, tais como água potável e saneamento, nutrição, pobreza, condições de trabalho, clima, ou acesso a atendimento de saúde. O ambiente pode ser mudado para melhorar a saúde, evitar incapacidades, e melhorar os resultados finais para as pessoas com deficiência ${ }^{4}$. 
Neste estudo, a dificuldade de acesso aos serviços de saúde nos diversos níveis de atenção foi fortemente associada com a presença de barreiras arquitetônicas. A presença de escadas, degraus altos, banheiros não adaptados, buracos e esgotos nas vias públicas constitui parte dos inúmeros exemplos que podem ser elencados ${ }^{39}$. A inadequação das calçadas e áreas próximas às USF, que devem ser a porta de entrada aos serviços de saúde, resulta da precariedade da infraestrutura e também interfere no acesso aos espaços destinados a prestação de serviços. Dados similares foram constatado em população maior que 18 anos de idade também na cidade de João Pessoa, uma vez que $63,9 \%$ das pessoas com deficiência informaram que as barreiras arquitetônicas eram obstáculos no acesso à saúde ${ }^{32}$.

Além disto, observa-se que muitas USF são frequentemente instaladas em prédios improvisados, que estavam disponíveis nas comunidades e não apresentam uma construção específica para atender as necessidades de todos os usuários e mesmo dos profissionais que lá trabalham ${ }^{40}$. Nesse sentido, Amaral et al. ${ }^{32}$ apontaram que 41,7\% da população com deficiência da cidade de João Pessoa relata não ter adaptações nos locais de atendimento. No entanto, é sabido que os projetos de urbanização das vias públicas, dos parques e demais espaços deveriam ser pensados e executados com o objetivo de viabilizar o direito de movimentar-se com segurança às pessoas idosas, com mobilidade reduzida ou com deficiências.

A dificuldade de acesso aos serviços de saúde também pode se dar devido ao distanciamento geográfico entre a residência e o serviço. Observou-se, em estudo prévio, que as pessoas com deficiência física e mobilidade reduzida de João Pessoa se deslocavam principalmente através de carros alugados, enquanto as pessoas com deficiência mental e auditiva iam preferencialmente a pé ou de transporte coletivo para suas consul$\operatorname{tas}^{32}$. A falta de meios de locomoção privado faz com que esta população dependa de transporte coletivo ou de ambulância, que não suportam toda a demanda dos que os procuram ${ }^{37}$. Em muitos destes transportes faltava adaptação apropriada para efetivar a locomoção destes usuários que possuíam limitações provenientes dos agravos à saúde.

Estudo sobre a razão da falta de assistência a saúde em países desenvolvidos apontam diferenças estatisticamente significativas para a falta de transporte, considerando a população deficiente $(30,3 \%)$ na faixa etária maior que 60 anos, quando comparado com a população não-deficiente
$(9,5 \%)^{13}$. Tal fato foi confirmado em nossa pesquisa, visto que a existência de um transporte adequado e adaptado para esta população foi considerado um fator de proteção para o acesso do usuário idoso e com deficiência aos serviços de saúde. Deste modo, mudanças em grande escala para melhorar a acessibilidade no sistema de transportes ou na infraestrutura de serviços públicos reduzirão essas barreiras ${ }^{4}$.

Observou-se que, dentre as limitações dos serviços de saúde, a ausência de atendimento prioritário $(38,9 \%)$ e o elevado tempo de espera para ser atendido $(27,9 \%)$ foram os mais citados pelos idosos. Sabe-se que esse fato fere o estatuto do idoso e a lei pioneira de nº4.737 de julho de 1965 , que garante a preferência nessas situações ${ }^{41}$. $\mathrm{Na}$ Europa, um estudo sobre os serviços de atendimento médico discutiu a importância de barreiras organizacionais para o acesso de pessoas com deficiência a tais serviços ${ }^{42}$. Dentre essas destacam-se: listas de espera, falta de um sistema de reservas para agendamentos, e complexos sistemas de referências. Estas barreiras são mais complicadas para pessoas com deficiência, por terem dificuldade de chegar cedo ou esperar o dia todo, ou por não conseguirem navegar em sistemas complexos ${ }^{42}$.

A falta de atendimento prioritário nos serviços de saúde gera como consequência uma longa espera para o atendimento. Tal fato pode causar desconfortos e dificuldades, principalmente com o avançar da idade para pessoas que apresentam limitações em suas atividades diárias, por ser preciso, muitas vezes, enfrentar filas durante horas para conseguir atendimento para consultas médicas ${ }^{23}$. Por conseguinte, a prontidão para ser atendido poderia proporcionar o melhor acesso à saúde e com uma melhor qualidade para o idoso com deficiência. Esse fato foi verídico para nosso estudo que evidenciou que a rapidez em ser atendido foi um fator de proteção na dificuldade de acesso aos serviços de saúde.

A falta de atendimento domiciliar (21,3\%) pode representar para a população estudada uma dificuldade para os cuidados de saúde, uma vez que esta é uma ferramenta importante na assistência ao idoso com deficiência. Além de muitos idosos encontrarem dificuldades em se deslocar até a USF, o atendimento em domicílio facilitaria o acompanhamento das condições de saúde, evitando o aparecimento de complicações secundárias à deficiência ${ }^{38}$. A importância desta prática também é confirmada pelo fato do idoso, muitas vezes, necessitar de um acompanhante nas consultas realizadas. 
No entanto, observou-se que a maioria dos entrevistados $(91,0 \%)$ recebia visitas regulares do Agente Comunitário de Saúde (ACS). Sabe-se que este profissional tem participação essencial no exercício da prevenção de doenças e promoção de saúde, mediante ações domiciliares ou comunitárias, individuais ou coletivas desenvolvidas em conformidade com as diretrizes do SUS e sob supervisão de gestor local. O presente estudo constatou que a inexistência da visita domiciliar do ACS aumentava em aproximadamente três vezes a chance de ter dificuldade no acesso aos serviços de saúde, confirmando a importância deste profissional no processo.

Pôde-se observar também, que a dificuldade no acesso à saúde foi fortemente associada à marcação de consultas médicas. Acredita-se que o desequilíbrio encontrado entre a grande demanda de usuários do SUS e a diminuta equipe contratada das USF pode provocar atrasos nas marcações de consultas, adiando tratamentos e intervenções que podem trazer riscos a vida. É sabido que a demora na marcação de consultas pode levar muitas famílias a recorrerem às consultas particulares. Porém, pessoas com baixo nível socioeconômico tendem a agravarem seu estado de saúde, por não possuírem condições de recorrer ao plano privado ou pagamento de consultas, visto que o diagnóstico e a consulta com especialistas são primordiais na garantia da saúde do idoso que possui deficiência ${ }^{38}$.

O somatório dos fatores expostos mostra que as barreiras arquitetônicas e a situação atual de atenção à saúde precisam ser adequadas vi- sando o ingresso e a utilização plena dos idosos com deficiência aos serviços de saúde.

\section{Conclusão}

$\mathrm{O}$ acelerado processo de envelhecimento populacional, marcado pelos agravos provocados pelas doenças crônico-degenerativas, baixa escolaridade e renda torna-se um problema de saúde pública a ser reconhecido para a população de João Pessoa. Enfatiza-se a necessidade de reformulação do cuidado ao idoso e reorganização dos serviços de saúde, visto que o sistema precisa se adequar melhor para comportar essa nova realidade da pirâmide etária.

O contingente idoso recebe tutela constitucional na garantia de seus direitos, sendo primordial que o governo assegure o acesso dos idosos aos sistemas de saúde. Fatores como a ausência de barreiras arquitetônicas, existência de transporte adaptados e de boa qualidade, facilidades de marcação de consultas com baixo tempo de espera são necessários para viabilizar o acesso à saúde.

Investimentos ou ações públicas devem ser intensificados para dar continuidade e otimizar a rede de serviços em saúde, e assim, comportar satisfatoriamente a demanda crescente de indivíduos idosos, principalmente com deficiência. Além disso, especial atenção deve ser dada aos jovens de hoje e idosos do futuro, para que com a elevada expectativa de vida tenhamos futuramente idosos mais saudáveis, sem desenvolverem deficiências consequentes de doenças crônicas.

\section{Colaboradores}

FLJS Amaral, MHA Motta, LPG Silva e SB Alves contribuíram na concepção e no delineamento ou na análise e interpretação dos dados; redação do artigo ou na sua revisão crítica; e aprovação da versão a ser publicada. 


\section{Referências}

1. Maciel ACC, Guerra RO. Fatores associados à alteração da mobilidade em idosos residentes na comunidade. Rev Bras Fisioter 2005; 9(1):17-23.

2. Brasil. Envelhecimento e saúde da pessoa idosa. Brasília: Ministério da Saúde; 2006.

3. Carvalho Filho ET, Papaléo Neto M. Geriatria: Fundamentos, Clínica e Terapêutica. 2a Edição. São Paulo: Ed. Atheneu; 2006.

4. Organização Mundial da Saúde (OMS). Relatório mundial sobre a deficiência. São Paulo: SEDPcD; 2012.

5. Brasil. Decreto 3.298 de 20 de Dezembro de 1999 Dispõe sobre a Política Nacional para a Integração da Pessoa Portadora de Deficiência, consolida as normas de proteção, e dá outras providências. Diário Oficial da União 1999; $21 \mathrm{dez}$.

6. Brasil. Decreto 186 de 09 de Julho de 2008. Aprova o texto da Convenção sobre os Direitos das Pessoas com Deficiência e de seu Protocolo. Diário Oficial da União 2008; 9 jul.

7. Castro SS, Lefevre F, Lefevre AMC, Cesar CLG. Acessibilidade aos serviços de saúde por pessoas com deficiência. Rev Saude Publica 2011; 45(1):99-105.

8. Medeiros M, Diniz D. Envelhecimento e deficiência. In: Camarano AA, organizador. Os Novos Idosos Brasileiros: Muito Além dos 60? Rio de Janeiro: IPEA; 2004.

9. Szwarcwald CL, Mendoça MHM, Andrade CLT. Indicadores de atenção básica em quatro municípios do Estado do Rio de Janeiro, 2005: resultados de inquérito domiciliar de base populacional. Cien Saude Colet 2006, 11(3):643-655.

10. Instituto Brasileiro de Estatística e Geografia (IBGE). Censo demográfico 2000. [site na Internet]. [acessado 2012 abr 06]. Disponível em: http://www.ibge. gov.br.

11. Frenk J. El concepto y la medición de accesibilidad. Salud Publica Mex 1985; 27:438-453.

12. Ferri CP, Acosta D, Guerra M, Huang Y, LlibreRodriguez JJ, Salas A, Sosa AL, Williams JD, Gaona C, Liu Z, Noriega-Fernandez L, Jotheeswaran AT, Prince1 MJ. Socioeconomic Factors and All Cause and Cause-Specific Mortality among Older People in Latin America, India, and China: A PopulationBased Cohort Study. PLoS Medicine 2012; 9(2):e1001179.
13. Coleridge P, Simonnot C, Steverlynck D. Study of disability in EC Development Cooperation. European Commission; 2010 [site na Internet]. [acessado 2012 abr 07]. Disponível em: http://www.who.int/nmh/ a5817/en/.

14. Almeida ES, Castro CGJ, Lisboa CA. Distritos Sanitários: Concepção e Organização. São Paulo: Faculdade de Saúde Pública da Universidade de São Paulo; 1998.

15. Brasil. Ministério da Saúde (MS). Conselho Nacional de Saúde. Resolução no ${ }^{\circ}$ 196/1996. Diretrizes e Normas Regulamentadoras de Pesquisas Envolvendo Seres Humanos. Diário Oficial da União 1996; out 10 .

16. Caldas PC. Envelhecimento com dependência: responsabilidades e demandas da família. Cad Saude Publica 2003; 19(3):773-781.

17. Nasri F.O envelhecimento populacional no Brasil. Einstein 2008; 6(Supl.):S4-S6.

18. Bos AMG, Bos AJG. Determinantes na escolha entre atendimento de saúde privada e pública por idosos. Rev Saude Publica 2004; 38(1):113-120.

19. Feliciano AB, Moraes AS, Freitas ICM. O perfil do idoso de baixa renda no Município de São Carlos, São Paulo, Brasil: um estudo epidemiológico. Cad Saude Publica 2004; 20(6):1575-1585.

20. Garcia ESS, Saintrain MVL. Perfil epidemiológico de uma população idosa atendida pelo programa saúde da família. Rev Enferm 2009; 17(1):18-23.

21. Lima, CVL, Bueno, CMLB. Envelhecimento e gênero: a vulnerabilidade de idosas no Brasil. Saúde Pesq 2009; 2(2):273-280.

22. Carboni RM, Reppetto MA. Uma reflexão sobre a assistência à saúde do idoso no Brasil. Rev Eletrônica de Enfermagem 2007; 9(1):251-260.

23. Lima-Costa MF, Barrero S, Giatti L, Uchôa E. Desigualdade social e saúde entre idosos brasileiros: um estudo baseado na Pesquisa Nacional por Amostra de Domicílios. Cad Saude Publica 2003; 19(3):745757.

24. Ramos LR. Fatores determinantes do envelhecimento saudável em idosos residentes em centro urbano: Projeto Epidoso, São Paulo. Cad Saude Publica 2003; 19(3):793-798. 
25. Beatty PW, Hagglund KJ, Neri MT, Dhont KR, Clark MJ, Hilton SA. Access to health care services among people with chronic or disabling conditions: patterns and predictors. Arch Phys Med Rehabil 2003; 84(10):1417-1425.

26. VanLeit B, Rithy P, Channa S. Secondary prevention of disabilities in the Cambodian provinces of Siem Reap and Takeo: perceptions of and use of the health system to address health conditions associated with disability in children. Brussels: Handicap International; 2007.

27. Bowers B, Lutz B, Esmond S, Jacobson N. Improving primary care for persons with disabilities: the nature of expertise. Disabil Soc 2003; 18(4):443-455.

28. Gulley SP, Altman BM. Disability in two health care systems: access, quality, satisfaction, and physician contacts among working-age Canadians and Americans with disabilities. Disabil Health J 2008; 1(4):196208.

29. Paiva KM, Cesar CLG, Alves MCGP, Barros MBA, Carandina L, Goldbaum M. Envelhecimento e deficiência auditiva referida. Cad Saude Publica 2011, 27(7):1292-1300.

30. Girondi JBR, Santos SMA. Deficiência física em idosos e acessibilidade na atenção básica em saúde: revisão integrativa da literatura. Rev Gaúcha Enferm 2011; 32(2):378-384.

31. Tannure MC, Alves M, Sena RR, Chianca TCM. Perfil epidemiológico da população idosa de Belo Horizonte, MG, Brasil. Rev Bras Enferm 2010; 63(5):817-822.

32. Amaral FLJS; Holanda CMA, Quirino MAB, Nascimento JPS, Neves RF, Ribeiro KSQS, Alves SB. Acessibilidade de pessoas com deficiência ou restrição permanente de mobilidade ao SUS. Cien Saude Colet 2012; 17(7):1833-1840.

33. Neves LF, Chen SR. Atenção à saúde do idoso com deficiência. São Paulo: Secretaria Municipal de Saúde; 2002. [site na Internet]. [acessado 2012 abr 06]. Disponível em: http://ww2.prefeitura.sp.gov.br// arquivos/secretarias/saude/deficiencia/0010/Saude_ idoso_com_deficiencia.pdf.
34. Coelho CLM, Bastos CL, Camara FP, Landeira-Fernandez J. A influência do gênero e da escolaridade no diagnóstico de demência. Estud Psicol 2010; 27(4): 449-456.

35. Megale RL, Iório MCM, Schochat E. Treinamento auditivo: avaliação do benefício em idosos usuários de próteses auditivas. Pró-Fono Rev At Ci 2010; 22(2):101-106.

36. Bortolon PC. Internações do SUS para fratura osteoporótica de fêmur em idosos. Cad Saude Publica 2011; 27(4):733-742.

37. Organização Mundial da Saúde (OMS). CIF: Classificação Internacional de Funcionalidade, Incapacidade e Saúde. São Paulo: Editora da Universidade de São Paulo; 2003.

38. Carreira L, Rodrigues RAP. Dificuldades dos familiares de idosos portadores de doenças crônicas no acesso à Unidade Básica de Saúde. Rev Bras Enferm 2010; 63(6):933-939.

39. Siqueira FCV, Facchini LA, Silveira DS, Piccini RX, Thumé E, Tomasi E. Barreiras arquitetônicas a idosos e portadores de deficiência física: um estudo epidemiológico da estrutura física das unidades básicas de saúde em sete estados do Brasil. Cien Saude Colet 2009; 14(1):39-44.

40. Facchini LA, Piccini RX, Tomasi E, Thumé E, Silveira DS, Siqueira FV, Rodrigues MAP. Desempenho do PSF no Sul e no Nordeste do Brasil: avaliação institucional e epidemiológica da Atenção Básica a Saúde. Cien Saude Colet 2006; 11(3):669-681.

41. Moimaz SAS, Garbin CAS, Saliba NA, Lolli LF. O idoso no Brasil - aspectos legislativos de relevância para profissionais de saúde. Rev Espaç Saúde 2009; 10(2):61-69.

42. Scheer JM, Kroll T, Neri MT, Beatty P. Access barriers for persons with disabilities: the consumers perspective. J Disabil Pol Stud 2003; 13(4):221-230.

Artigo apresentado em 30/04/2012

Aprovado em 17/07/2012

Versão final apresentada em 15/08/2012 